\title{
EXTRINSIC UPPER BOUNDS FOR EIGENVALUES OF DIRAC-TYPE OPERATORS
}

\author{
N. ANGHEL \\ (Communicated by Peter Li)
}

\begin{abstract}
Extrinsic estimates from above for eigenvalues of generalized Dirac operators on compact manifolds are given. They depend on the second fundamental form of any isometric immersion of the manifold in some Euclidean space and the curvature term in the Bochner-Weitzenböck formula for the square of the Dirac operator. Most of the known extrinsic upper bounds for the first eigenvalue of the Laplacian are in this way easily recovered and extended.
\end{abstract}

\section{INTRODUCTION}

Let $M$ be a compact connected $n$-dimensional Riemannian manifold isometrically immersed in some $\mathbf{R}^{n}$.

This paper presents an attempt to unify partially various methods of finding extrinsic estimates for eigenvalues of the Laplace operator $\Delta$ on $M$ [BW, C, $\mathrm{Li}, \mathrm{R}, \mathrm{YY}$ ], or the Laplace-Beltrami operator on forms [L], and extend them to the class of generalized Dirac operators defined in [GL, LM]. The extrinsic character of the estimates is reflected in their dependence on the geometry of the immersion chosen.

The method of proof was originated by Payne, Pólya, and Weinberger in [PPW] and can also be found in many of the papers cited above. It consists of constructing test sections for the Rayleigh's principle out of the components of the immersion and the eigensections corresponding to various eigenvalues of the operator. The machinery of generalized Dirac operators makes the whole analysis simple, economical, and elegant.

In order to convey some idea of the kind of results we obtained, consider the following example (see Corollary 3.8 below):

The square of the smallest, in absolute value, nonzero eigenvalue of the classical Dirac operator on an even-dimensional spin manifold with nonvanishing

Received by the editors November 28, 1990 and, in revised form, June 10, 1991; presented on January 17, 1991 at the AMS Annual Meeting \#863, January 16-19, 1991 in San Francisco, CA.

1991 Mathematics Subject Classification. Primary 53C20, 58G25.

Key words and phrases. Isometric immersion, generalized Dirac operator, eigenvalue, upper bound.

Partially supported by UNT Grant No. 34661 . 
$\widehat{A}$-genus is bounded above by $\sup _{M}|h|^{2} / \operatorname{dim} M$, where $h$ is the second fundamental form of $M$ in $\mathbf{R}^{\bar{n}}$.

\section{Preliminaries}

Let $x=\left(x^{1}, \ldots, x^{n}\right): M \rightarrow \mathbf{R}^{n}$ be an isometric immersion of $M$ in $\mathbf{R}^{n}$. The existence of $x$ is guaranteed by the Nash embedding theorem. One concludes immediately that, pointwise on $M$,

$$
\sum_{\alpha=1}^{n}\left|\operatorname{grad} x^{\alpha}\right|^{2}=n
$$

Let $\nabla$ and $\bar{\nabla}$ be the covariant differentiations on $M$ and $\mathbf{R}^{\bar{n}}$ respectively. If $X$ and $Y$ are vector fields tangent to $M$, the second fundamental form $h(X, Y)$ of $M$ in $\mathbf{R}^{n}$ is defined by the equation of Gauss

$$
\bar{\nabla}_{X} Y=\nabla_{X} Y+h(X, Y) \text {, }
$$

where $h$ is a normal bundle-valued symmetric 2-tensor on $M$. In particular, if $Z$ is a third vector field tangent to $M$,

$$
h(X, Y) \perp d x(Z) .
$$

The mean curvature vector field $\eta$ is defined by $\eta=\operatorname{tr} h=\sum_{i=1}^{n} h\left(e_{i}, e_{i}\right)$, where $\left(e_{i}\right)_{i}$ is a local orthonormal frame in $T M$. One has [KN]

$$
\eta=-\left(\Delta x^{1}, \ldots, \Delta x^{n}\right) \text {. }
$$

Finally $H=\frac{1}{n}|\eta|$ is the mean curvature function.

Let $\mathrm{Cl}(M)$ be the Clifford bundle of algebras induced by the tangent bundle $T M$ and the Riemannian metric $\langle$,$\rangle [GL, LM]. There is a canonical embed-$ ding $T M \hookrightarrow \mathrm{Cl}(M)$ and then metric and covariant differentiation extend from $T M$ to $\mathrm{Cl}(M)$ in such a way that the covariant differentiation $\nabla$ on $\mathrm{Cl}(M)$ preserves the metric and acts as a derivation. There is a canonical vector bundle isomorphism $\mathrm{Cl}(M) \cong \Lambda^{*} M$.

A bundle of left modules $S \rightarrow M$ over $\mathrm{Cl}(M)$ is a generalized Dirac bundle if $S$ is furnished with a Hermitian metric $\langle$,$\rangle and a metric connection \nabla$ such that

$$
\text { unit vectors in } T M \subset \mathrm{Cl}(M) \text { act isometrically on } S \text {, }
$$

$$
\nabla_{X}(\phi s)=\nabla_{X}(\phi) s+\phi \nabla_{X} s
$$

for any vector field $X$ tangent to $M$ and any $\phi$ and $s$ smooth sections of $\mathrm{Cl}(M)$ and $S$ respectively.

Any generalized Dirac bundle generates a first-order elliptic differential operator $D: C^{\infty}(S) \rightarrow C^{\infty}(S)$, the generalized Dirac operator, which locally can be expressed as

$$
D=\sum_{i=1}^{n} e_{i} \nabla_{e_{i}}
$$

Equalities (1.5) and (1.4b) readily imply, for $f \in C^{\infty}(M)$ and $s \in C^{\infty}(S)$,

$$
\begin{gathered}
D(f s)=(\operatorname{grad} f) s+f D s, \\
D^{2}(f s)=\Delta(f) s-2 \nabla_{\operatorname{grad} f} s+f D^{2} s .
\end{gathered}
$$


$D$ is selfadjoint with respect to the usual inner product $\left(s_{1}, s_{2}\right)=$ $\int_{M}\left\langle s_{1}, s_{2}\right\rangle$ in $C^{\infty}(S)$.

For $D^{2}$ one has the following Bochner-Weitzenböck formula [GL, LM]:

$$
D^{2}=\nabla^{*} \nabla+\mathscr{R} \text {. }
$$

In (1.7) $\mathscr{R}$ is a Hermitian curvature bundle morphism acting on $S$ according to the formula $\mathscr{R}=\sum_{i<j} e_{i} e_{j} R_{e_{i}, e_{j}}, R_{e_{i}, e_{j}}=\left[\nabla_{e_{i}}, \nabla_{e_{j}}\right]-\nabla_{\left[e_{i}, e_{j}\right]}$.

The standard examples of generalized Dirac bundles and operators are

(1.8) $S=\mathrm{Cl}(M)$. Under the isomorphism $\mathrm{Cl}(M) \cong \Lambda^{*}(M)$, one has $D \cong$ $d+d^{*}$, the Gauss-Bonnet operator, $D^{2} \cong d^{*} d+d d^{*}$, the Laplace-Beltrami operator on forms, and $\mathscr{R}_{\mid M \times C}=0, \mathscr{R}_{\mid T M}=$ Ric, the Ricci transformation.

(1.9) $M$ spin manifold, $S=\Sigma$, the spinor bundle. Then $D$ is the classical Dirac operator and $\mathscr{R}=\frac{1}{4} k, k$ the scalar curvature function on $M$.

\section{Main estimate}

Let $E$ be a subbundle of $S$ invariant under $\nabla$ and $\mathscr{R}$, i.e., $\nabla_{X}\left(C^{\infty}(E)\right)$ $\subset C^{\infty}(E)$, for any vector field $X$, and $\mathscr{R}(E) \subset E$ respectively. Then $C^{\infty}(E)$ is $D^{2}$-invariant, by the Bochner-Weitzenböck formula (1.7). The restriction of $D^{2}$ to $C^{\infty}(E)$ has a discrete spectrum located on the nonnegative part of the real line. Its eigenvalues can be arranged, counting multiplicities, in an increasing sequence

$$
0 \leq \lambda_{1} \leq \lambda_{2} \leq \cdots
$$

Eigensections $s_{1}, s_{2}, \ldots, s_{l}, \ldots, D^{2} s_{l}=\lambda_{l} s_{l}$ can be chosen in $C^{\infty}(E)$ to form an orthonormal basis for $L^{2}(E)$, the completion of $C^{\infty}(E)$ with respect to the global inner product $($,$) . We want to estimate any given eigenvalue \lambda_{m+1}$ in terms of the previous ones, $\lambda_{1}, \lambda_{2}, \ldots, \lambda_{m}$, their eigensections $s_{1}, s_{2}, \ldots, s_{m}$, the immersion $x$, and the curvature term $\mathscr{R}$.

Theorem 2.1. Let $M$ be a compact Riemannian manifold of dimension $n$ and $x: M \rightarrow \mathbf{R}^{n}$ be an isometric immersion with mean curvature function $H$. Let $\left(\lambda_{1}, s_{1}\right),\left(\lambda_{2}, s_{2}\right), \ldots, 0 \leq \lambda_{1} \leq \lambda_{2} \leq \cdots, s_{l} \in C^{\infty}(E)$ be the spectral decomposition of $D^{2}=\nabla^{*} \nabla+\mathscr{R}: C^{\infty}(E) \rightarrow C^{\infty}(E)$, the restriction of the square of a generalized Dirac operator $D: C^{\infty}(S) \rightarrow C^{\infty}(S)$ to some $\nabla$-invariant, $\mathscr{R}$ invariant subbundle $E$ of $S$. Then

$$
\lambda_{m+1}-\lambda_{m} \leq \frac{n}{m} \sum_{l=1}^{m}\left(H^{2} s_{l}, s_{l}\right)+\frac{4}{m n} \sum_{l=1}^{m} \lambda_{l}-\frac{4}{m n} \sum_{l=1}^{m}\left(\mathscr{R} s_{l}, s_{l}\right) .
$$

Proof. For $1 \leq l \leq m$ and $f \in C^{\infty}(M), f$ real-valued, define $s_{l}^{f} \in C^{\infty}(E)$ by

$$
s_{l}^{f}:=f s_{l}-\sum_{k=1}^{m}\left(f s_{l}, s_{k}\right) s_{k} .
$$

By construction the section $s_{l}^{f}$ is $L^{2}$-orthogonal to $s_{1}, s_{2}, \ldots, s_{m}$, so by the Rayleigh's principle

$$
\lambda_{m+1}\left(s_{l}^{f}, s_{l}^{f}\right) \leq\left(D^{2} s_{l}^{f}, s_{l}^{f}\right)
$$


On one hand, using (1.6b)

$$
\begin{aligned}
D^{2} s_{l}^{f} & =D^{2}\left(f s_{l}\right)-\sum_{k=1}^{m}\left(f s_{l}, s_{k}\right) \lambda_{k} s_{k} \\
& =\Delta(f) s_{l}-2 \nabla_{\operatorname{grad} f} s_{l}+\lambda_{l} f s_{l}-\sum_{k=1}^{m} \lambda_{k}\left(f s_{l}, s_{k}\right) s_{k} .
\end{aligned}
$$

From the orthogonality of $s_{l}^{f}$ on $\operatorname{span}\left\{s_{1}, \ldots, s_{m}\right\}$ and the equation above, we get

$$
\left(D^{2} s_{l}^{f}, s_{l}^{f}\right)-\lambda_{l}\left(s_{l}^{f}, s_{l}^{f}\right)=\left(\Delta(f) s_{l}-2 \nabla_{\operatorname{grad} f} s_{l}, s_{l}^{f}\right) .
$$

On the other hand,

$$
\begin{aligned}
\left(D^{2} s_{l}^{f}, s_{l}^{f}\right)-\lambda_{l}\left(s_{l}^{f}, s_{l}^{f}\right) & =\left(D^{2}\left(f s_{l}\right)-f D^{2} s_{l}, s_{l}^{f}\right) \\
& =\left(D^{2}\left(f s_{l}\right)-f D^{2} s_{l}, f s_{l}\right)-\left(D^{2}\left(f s_{l}\right)-f D^{2} s_{l}, \sigma_{f l}\right),
\end{aligned}
$$

where $\sigma_{f l}=\sum_{k=1}^{m}\left(f_{l}, s_{k}\right) s_{k}$. Notice now that the quantities

$$
\left(D^{2}\left(f s_{l}\right)-f D^{2} s_{l}, f s_{l}\right) \quad \text { and } \quad\left(D^{2}\left(f s_{l}\right)-f D^{2} s_{l}, \sigma_{f l}\right)
$$

must be real. Also

$$
\begin{aligned}
\left(D^{2}(\right. & \left.\left.f_{s_{l}}\right)-f D^{2} s_{l}, f s_{l}\right) \\
= & \left(D\left(f s_{l}\right), D\left(f s_{l}\right)\right)-\left(D s_{l}, D\left(f^{2} s_{l}\right)\right) \\
\stackrel{1.6 a}{=} & \left(\operatorname{grad} f s_{l}+f D s_{l}, \operatorname{grad} f s_{l}+f D s_{l}\right)-\left(D s_{l}, 2 f \operatorname{grad} f s_{l}+f^{2} D s_{l}\right) \\
= & \left(|\operatorname{grad} f|^{2} s_{l}, s_{l}\right)+\left(f D s_{l}, \operatorname{grad} f s_{l}\right)+\left(\operatorname{grad} f s_{l}, f D s_{l}\right) \\
& +\left(D s_{l}, f^{2} D s_{l}\right)-2\left(f D s_{l}, \operatorname{grad} f s_{l}\right)-\left(D s_{l}, f^{2} D s_{l}\right) \\
= & \left(|\operatorname{grad} f|^{2} s_{l}, s_{l}\right)+\left(\operatorname{grad} f s_{l}, f D s_{l}\right)-\overline{\left(\operatorname{grad} f s_{l}, f D s_{l}\right)} \\
= & \left(|\operatorname{grad} f|^{2} s_{l}, s_{l}\right)
\end{aligned}
$$

since ( $\left.\operatorname{grad} f s_{l}, f D s_{l}\right)-\overline{\left(\operatorname{grad} f s_{l}, f D s_{l}\right)}$ must vanish as the only purely imaginary quantity in an equation involving only real quantities otherwise. Thus

$$
\left(D^{2}\left(f s_{l}\right)-f D^{2} s_{l}, f s_{l}\right)=\left(|\operatorname{grad} f|^{2} s_{l}, s_{l}\right) .
$$

Arguing in a similar way we also have

$$
\sum_{l=1}^{m}\left(D^{2}\left(f s_{l}\right)-f D^{2} s_{l}, \sigma_{f l}\right)=0 .
$$

Now let $f=x^{\alpha}, \alpha=1, \ldots, \bar{n}$, and $s_{\alpha l}:=s_{l}^{x^{\alpha}}$. Then (2.3), (2.4), (2.5), and (1.1) immediately yield

$$
\sum_{l=1}^{m} \sum_{\alpha=1}^{n}\left(\Delta\left(x^{\alpha}\right) s_{l}-2 \nabla_{\operatorname{grad} x^{\alpha}} s_{l}, s_{\alpha l}\right)=m n .
$$


In particular, equation (2.6) implies that $s_{\alpha l} \neq 0$ for some $(\alpha, l)$. Since for $l \leq m, \lambda_{l} \leq \lambda_{m}$, letting $f=x^{\alpha}, s_{l}^{f}=s_{\alpha l}$ and summing over $l$ and $\alpha$ in equation (2.3) one gets, via equation (2.6),

$$
\sum_{l=1}^{m} \sum_{\alpha=1}^{\bar{n}}\left(D^{2} s_{\alpha l}, s_{\alpha l}\right)-\lambda_{m} \sum_{l=1}^{m} \sum_{\alpha=1}^{\bar{n}}\left(s_{\alpha l}, s_{\alpha l}\right) \leq m n .
$$

This inequality and (2.2) then yield

$$
\left(\lambda_{m+1}-\lambda_{m}\right) \sum_{l=1}^{m} \sum_{\alpha=1}^{\bar{n}}\left(s_{\alpha l}, s_{\alpha l}\right) \leq m n .
$$

However from equation (2.6),

$$
\begin{aligned}
m^{2} n^{2} & =\left[\sum_{l=1}^{m} \sum_{\alpha=1}^{n}\left(\Delta\left(x^{\alpha}\right) s_{l}-2 \nabla_{\operatorname{grad} x^{\alpha}} s_{l}, s_{\alpha l}\right)\right]^{2} \\
& \leq\left[\sum_{l=1}^{m} \sum_{\alpha=1}^{n} \|\left(\Delta\left(x^{\alpha}\right) s_{l}-2 \nabla_{\operatorname{grad} x^{\alpha}} s_{l}\left\|_{L^{2}}\right\| s_{\alpha l} \|_{L^{2}}\right]^{2}\right. \\
& \leq \sum_{l=1}^{m} \sum_{\alpha=1}^{n}\left(\Delta\left(x^{\alpha}\right) s_{l}-2 \nabla_{\operatorname{grad} x^{\alpha}} s_{l}, \Delta\left(x^{\alpha}\right) s_{l}-2 \nabla_{\operatorname{grad} x^{\alpha}} s_{l}\right) \sum_{l=1}^{m} \sum_{\alpha=1}^{n}\left(s_{\alpha l}, s_{\alpha l}\right),
\end{aligned}
$$

and so equation (2.6) implies, since $\sum_{l=1}^{m} \sum_{\alpha=1}^{\bar{n}}\left(s_{\alpha l}, s_{\alpha l}\right) \neq 0$,

$$
\lambda_{m+1}-\lambda_{m} \leq \frac{1}{m n} \sum_{l=1}^{m} \sum_{\alpha=1}^{n}\left(\Delta\left(x^{\alpha}\right) s_{l}-2 \nabla_{\operatorname{grad} x^{\alpha}} s_{l}, \Delta\left(x^{\alpha}\right) s_{l}-2 \nabla_{\operatorname{grad} x^{\alpha}} s_{l}\right) .
$$

Theorem 2.1 follows now from inequality (2.8) and the lemma below.

\section{Lemma 2.9.}

$$
\begin{gathered}
\sum_{l=1}^{m} \sum_{\alpha=1}^{n}\left(\Delta\left(x^{\alpha}\right) s_{l}-2 \nabla_{\operatorname{grad} x^{\alpha}} s_{l}, \Delta\left(x^{\alpha}\right) s_{l}-2 \nabla_{\operatorname{grad} x^{\alpha}} s_{l}\right) \\
=n^{2} \sum_{l=1}^{m}\left(H^{2} s_{l}, s_{l}\right)+4 \sum_{l=1}^{m} \lambda_{l}-4 \sum_{l=1}^{m}\left(\mathscr{R} s_{l}, s_{l}\right)
\end{gathered}
$$

Proof.

$$
\begin{aligned}
\left(\Delta\left(x^{\alpha}\right)\right. & \left.s_{l}-2 \nabla_{\operatorname{grad} x^{\alpha}} s_{l}, \Delta\left(x^{\alpha}\right) s_{l}-2 \nabla_{\operatorname{grad} x^{\alpha}} s_{l}\right) \\
= & \left(\left(\Delta\left(x^{\alpha}\right)\right)^{2} x_{l}, x_{l}\right)-2\left[\left(\nabla_{\Delta\left(x^{\alpha}\right) \operatorname{grad} x^{\alpha}} s_{l}, s_{l}\right)+\left(s_{l}, \nabla_{\Delta\left(x^{\alpha}\right) \operatorname{grad} x^{\alpha}} s_{l}\right)\right] \\
& +4\left(\nabla_{\operatorname{grad} x^{\alpha}} s_{l}, \nabla_{\operatorname{grad} x^{\alpha}} s_{l}\right) \\
& =\left(\left(\Delta\left(x^{\alpha}\right)\right)^{2} s_{l}, s_{l}\right)-2 \int_{M} \Delta\left(x^{\alpha}\right) \operatorname{grad} x^{\alpha}\left(\left\langle s_{l}, s_{l}\right\rangle\right)+4\left(\nabla_{\operatorname{grad} x^{\alpha}} s_{l}, \nabla_{\operatorname{grad} x^{\alpha}} s_{l}\right) .
\end{aligned}
$$


Thus

$$
\begin{aligned}
\sum_{l=1}^{m} \sum_{\alpha=1}^{n}\left(\Delta\left(x^{\alpha}\right) s_{l}-2 \nabla_{\operatorname{grad} x^{\alpha}} s_{l}, \Delta\left(x^{\alpha}\right) s_{l}-2 \nabla_{\operatorname{grad} x^{\alpha}} s_{l}\right) \\
=\sum_{l=1}^{m} \sum_{\alpha=1}^{\bar{n}}\left(\left(\Delta\left(x^{\alpha}\right)\right)^{2} s_{l}, s_{l}\right)-2 \int_{M}\left(\sum_{\alpha=1}^{\bar{n}} \Delta\left(x^{\alpha}\right) \operatorname{grad} x^{\alpha}\right)\left(\sum_{l=1}^{m}\left\langle s_{l}, s_{l}\right\rangle\right) \\
\quad+4 \sum_{l=1}^{m} \sum_{\alpha=1}^{n}\left(\nabla_{\operatorname{grad} x^{\alpha}} s_{l}, \nabla_{\operatorname{grad} x^{\alpha}} s_{l}\right) \\
=n^{2} \sum_{l=1}^{m}\left(H^{2} s_{l}, s_{l}\right)-2 \int_{M}\left(\sum_{\alpha=1}^{n} \Delta\left(x^{\alpha}\right) \operatorname{grad} x^{\alpha}\right)\left(\sum_{l=1}^{m}\left\langle s_{l}, s_{l}\right\rangle\right) \\
\quad+4 \sum_{l=1}^{m} \sum_{\alpha=1}^{n}\left(\nabla_{\operatorname{grad} x^{\alpha}} s_{l}, \nabla_{\operatorname{grad} x^{\alpha}} s_{l}\right) .
\end{aligned}
$$

We claim that the vector field $\sum_{\alpha=1}^{n} \Delta\left(x^{\alpha}\right) \operatorname{grad} x^{\alpha}$ vanishes identically. Locally,

$$
\begin{aligned}
\sum_{\alpha=1}^{n} \Delta\left(x^{\alpha}\right) \operatorname{grad} x^{\alpha} & \sum_{\alpha=1}^{n} \sum_{i=1}^{n} \Delta\left(x^{\alpha}\right)\left\langle\operatorname{grad} x^{\alpha}, e_{i}\right\rangle e_{i} \\
& =\sum_{i=1}^{n}\left(\sum_{\alpha=1}^{n} \Delta\left(x^{\alpha}\right) e_{i}\left(x^{\alpha}\right)\right) e_{i}=-\sum_{i=1}^{n}\left\langle\eta, d x\left(e_{i}\right)\right\rangle e_{i}=0,
\end{aligned}
$$

by (1.2). Also

$$
\begin{gathered}
\sum_{\alpha=1}^{\bar{n}}\left\langle\nabla_{\operatorname{grad} x^{\alpha}} s_{l}, \nabla_{\operatorname{grad} x^{\alpha}} s_{l}\right\rangle=\sum_{\alpha=1}^{n} \sum_{i, j=1}^{n} e_{i}\left(x^{\alpha}\right) e_{j}\left(x^{\alpha}\right)\left\langle\nabla_{e_{i}} s_{l}, \nabla_{e_{j}} s_{l}\right\rangle \\
=\sum_{i, j=1}^{n}\left(\sum_{\alpha=1}^{n} e_{i}\left(x^{\alpha}\right) e_{j}\left(x^{\alpha}\right)\right)\left\langle\nabla_{e_{i}} s_{l}, \nabla_{e_{j}} s_{l}\right\rangle=\sum_{i=1}^{n}\left\langle\nabla_{e_{i}} s_{l}, \nabla_{e_{i}} s_{l}\right\rangle \\
=\sum_{i=1}^{n}\left\langle e_{i}^{*} \otimes \nabla_{e_{i}} s_{l}, e_{i}^{*} \otimes \nabla_{e_{i}} s_{l}\right\rangle=\left\langle\nabla s_{l}, \nabla s_{l}\right\rangle .
\end{gathered}
$$

As a result

$$
\begin{aligned}
\sum_{l=1}^{m} \sum_{\alpha=1}^{n}\left(\nabla_{\operatorname{grad} x^{\alpha}} s_{l}, \nabla_{\operatorname{grad} x^{\alpha}} s_{l}\right) & =\sum_{l=1}^{m}\left(\nabla^{*} \nabla s_{l}, s_{l}\right)=\sum_{l=1}^{m}\left(D^{2} s_{l}, s_{l}\right)-\sum_{l=1}^{m}\left(\mathscr{R} s_{l}, s_{l}\right) \\
& =\sum_{l=1}^{m} \lambda_{l}-\sum_{l=1}^{m}\left(\mathscr{R} s_{l}, s_{l}\right) .
\end{aligned}
$$

Putting all the pieces together, the lemma follows. 


\section{Consequences}

Certainly Theorem 2.1 , as it stands, does not give a very useful estimate. Especially undesirable is the presence of $s_{l}$ 's in its right-hand side, however, it is its generality that counts. We will show in this section that for particular classes of operators, various interplays between the mean curvature function $H$, the eigenvalues $\lambda_{l}$, and the curvature endomorphism $\mathscr{R}$ occur, making the estimate interesting.

Theorem 3.1. The hypotheses being the same as in Theorem 2.1, if $\mathscr{R}_{\mid E} \geq$ cId, for some $c \in \mathbf{R}$, then one has

$$
\lambda_{m+1}-\lambda_{m} \leq n H_{\infty}^{2}+\frac{4}{m n} \sum_{l=1}^{m} \lambda_{l}-\frac{4}{n} c,
$$

where $H_{\infty}^{2}=\sup _{M} H^{2}$.

Proof. Since $s_{l}$ are unit vectors in $L^{2}(E)$ and $\langle\mathscr{R} s, s\rangle \geq c\langle s, s\rangle$, the assertion follows immediately from Theorem 2.1 . The best constant $c$ is the infimum over $M$ of the minimum eigenvalue function of $\mathscr{R}_{\mid E}$.

Theorem 3.1 represents the crude way to free the estimate in Theorem 2.1 of $s_{l}$. It improves the main result of [L], where $D=d+d^{*}$, the Gauss-Bonnet operator, $E=\Lambda^{p}(M)$, and $c=-\|\mathscr{R}\|,\|\mathscr{R}\|$ being the $L^{2}$-operator norm of $\mathscr{R}$ in $C^{\infty}(E)$.

Corollary 3.2. The hypotheses being those of Theorem 3.1, one has

$$
\lambda_{m+1} \leq\left(\frac{4}{n}+1\right)^{m} \lambda_{1}+\frac{n}{4}\left[\left(\frac{4}{n}+1\right)^{m}-1\right]\left(n H_{\infty}^{2}-\frac{4}{n} c\right) .
$$

Proof. Since $\lambda_{l} \leq \lambda_{m}$ for $l \leq m, \sum_{l=1}^{m} \lambda_{l} \leq m \lambda_{m}$. The estimate in Theorem 3.1 becomes

$$
\lambda_{m+1} \leq\left(\frac{4}{n}+1\right) \lambda_{m}+n H_{\infty}^{2}-\frac{4}{n} c .
$$

By iteration it yields the claim.

Remark 3.3. Assume now that the isometric immersion $x: M \rightarrow \mathbf{R}^{n}$ is in fact a minimal immersion of $M$ into the Euclidean unit sphere $\mathbf{S}^{n-1} \subset \mathbf{R}^{n}$. Then the coordinate functions $x^{1}, x^{2}, \ldots, x^{n}$ are eigenfunctions of the Laplacian $\Delta$ with eigenvalue $n=\operatorname{dim} M[\mathrm{YY}]$, and $\eta=-n\left(x^{1}, \ldots, x^{\bar{n}}\right)$ and $H \equiv 1$. Thus in the case of minimally immersed submanifolds into Euclidean spheres, Theorem 3.1 gives the estimate

$$
\lambda_{m+1}-\lambda_{m} \leq n+\frac{4}{m n} \sum_{l=1}^{m} \lambda_{l}-\frac{4}{n} c .
$$

Corollary 3.4. In addition to the hypotheses of Theorem 3.1 assume that 0 is an eigenvalue of $D^{2}$ on $C^{\infty}(E)$. Then the first nonzero eigenvalue $\lambda$ of $D^{2}$ on $C^{\infty}(E)$ satisfies

$$
\lambda \leq n H_{\infty}^{2}-\frac{4}{n} c .
$$

Proof. The proof is a simple consequence of Theorem 3.1, for $\lambda_{1}=\lambda_{2}=\cdots=$ $\lambda_{m}, \lambda_{m+1}=\lambda$. 
Remark 3.5. Since the actual size of the 0-eigenspace of $D^{2}$ does not appear in the estimate in Corollary 3.4 , one is tempted to conjecture its validity regardless the existence of 0-eigensections. It does not work, however, as the classical Dirac operator (1.9) on the $n$-dimensional unit sphere $\mathbf{S}^{n}, n \geq 3$, embedded in $\mathbf{R}^{n+1}$ shows. Here $H \equiv 1$ and $\mathscr{R} \equiv n(n-1) / 4=c$; thus one would expect $\lambda \leq 1$. On the other hand, from the Bochner-Weitzenböck formula, $\lambda>n(n-1) / 4$.

Assume now that $\mathscr{R}_{\mid E}=0$, and let $s$ be an eigensection of $D^{2}$ with eigenvalue 0 . The Bochner-Weitzenböck formula implies then $\nabla s=0$, i.e., $s$ is parallel. Since $\nabla$ preserves the metric, $\langle s, s\rangle$ is a constant function on $M$.

Theorem 3.6. In addition to the hypotheses of Theorem 2.1 assume that 0 is in the spectrum of $D^{2}$ on $C^{\infty}(E)$ and $\mathscr{R}_{\mid E}=0$. Then the first nonzero eigenvalue $\lambda$ of $D^{2}$ on $C^{\infty}(E)$ satisfies

$$
\lambda \leq \frac{n}{\operatorname{vol} M} \int_{M} H^{2}
$$

Proof. Let $s_{1}, s_{2}, \ldots, s_{m}$ be an orthonormal basis for the 0-eigenspace of $D^{2}$ on $C^{\infty}(E)$. As above $\left\langle s_{l}, s_{l}\right\rangle$ is a constant function on $M$ and since $\left(s_{l}, s_{l}\right)=$ $1,\left\langle s_{l}, s_{l}\right\rangle=1 /$ vol $M$. The claim follows now from Theorem 2.1 , since $\lambda_{m+1}=$ $\lambda,\left(H^{2} s_{l}, s_{l}\right)=\int_{M} H^{2}\left\langle s_{l}, s_{l}\right\rangle, \lambda_{l}=0$ for $1 \leq l \leq m$, and $\mathscr{R} s_{l}=0$.

The Laplace-Beltrami operator $d^{*} d+d d^{*}$ restricted to functions $(E=\mathbf{C})$ provides such an example. Here the 0-eigenspace is one-dimensional. The theorem in this case is due to Reilly [R].

Another example of this type is furnished by the Laplace-Beltrami operator restricted to one-forms, on a Ricci-flat manifold with nonvanishing first Betti number, e.g., a Ricci-flat torus or any other Ricci-flat closed surface of positive genus.

Let us concentrate now on estimating the eigenvalues of the classical Dirac operator on a spin manifold (1.8).

Theorem 3.7. If $M$ is a $n$-dimensional spin manifold isometrically immersed in some $\mathbf{R}^{n}$ and $0 \leq \lambda_{1} \leq \lambda_{2} \leq \cdots$ are the eigenvalues of the square of the classical Dirac operator, counted with multiplicities, then

$$
\lambda_{m+1}-\lambda_{m} \leq \frac{\sup _{M}|h|^{2}}{n}+\frac{4}{m n} \sum_{l=1}^{m} \lambda_{l}
$$

where $h$ is the second fundamental form of the immersion and $|h|^{2}=$ $\sum_{i, j}\left|h\left(e_{i}, e_{j}\right)\right|^{2}$.

Proof. In this case $\mathscr{R}=\frac{k}{4}, k$ scalar curvature; thus the estimate in Theorem 2.1 becomes

$$
\lambda_{m+1}-\lambda_{m} \leq \frac{1}{m n} \sum_{l=1}^{m}\left(\left(|\eta|^{2}-k\right) s_{l}, s_{l}\right)+\frac{4}{m n} \sum_{l=1}^{m} \lambda_{l} .
$$

However $|\eta|^{2}-k=|h|^{2}$, and the desired estimate follows. 
Corollary 3.8. Let $M$ be an even-dimensional spin manifold with nonvanishing $\widehat{A}$-genus. Then the smallest, in absolute value, nonzero eigenvalue $\lambda$ of the classical Dirac operator on $M$ satisfies

$$
\lambda^{2} \leq \sup _{M}|h|^{2} / \operatorname{dim} M
$$

where $h$ is the second fundamental form of any isometric immersion of $M$ in some Euclidean space.

Proof. By the Atiyah-Singer index theorem [LM, p. 256] a nonvanishing $\widehat{A}$ genus ensures the existence of harmonic spinors for the classical Dirac operator, i.e., 0-eigensections. Then the corollary follows from Theorem 3.7, letting $\lambda_{1}=$ $\lambda_{2}=\cdots=\lambda_{m}=0$ and $\lambda_{m+1}=\lambda^{2}$.

\section{REFERENCES}

[BW] D. D. Bleeker and J. L. Weiner, Extrinsic bounds on $\lambda_{1}$ of $\Delta$ on a compact manifold, Comment. Math. Helv. 51 (1976), 601-609.

[C] S.-Y. Cheng, Eigenfunctions and eigenvalues of Laplacian, Proc. Sympos. Pure Math., vol. 27, Amer. Math. Soc., Providence, RI, 1975, pp. 185-193.

[GL] M. Gromov and H. B. Lawson, Positive scalar curvature and the Dirac operator on complete Riemannian manifolds, Inst. Hautes Études Sci. Publ. Math. 58 (1983), 259-408.

[KN] S. Kobayashi and K. Nomizu, Foundations of differential geometry, Interscience, New York, London, and Sidney, 1969.

[L] J. Lee, The gaps in the spectrum of the Laplace-Beltrami operator, Houston J. Math. 17 (1991), 1-24.

[Li] P. Li, Eigenvalue estimates on homogeneous manifolds, Comment. Math. Helv. 55 (1980), 347-363.

[LM] H. B. Lawson and M.-L. Michelsohn, Spin geometry, Princeton Univ. Press, Princeton, NJ, 1989.

[PPW] L. E. Payne, G. Pólya, and H. F. Weinberger, On the ratio of consecutive eigenvalues, J. Math. Phys. 35 (1956), 289-298.

[R] R. C. Reilly, On the first eigenvalue of the Laplacian for compact submanifolds of Euclidean space, Comment. Math. Helv. 52 (1977), 525-533.

[YY] P. Yang and S. T. Yau, Eigenvalues of the Laplacian on compact Riemann surfaces and minimal submanifolds, Ann. Scuola. Norm. Sup. Pisa Cl. Sci. (4) 7 (1980), 55-63.

Department of Mathematics, University of North Texas, Denton, Texas 76203

E-mail address: anghel@vaxb.acs.unt.edu 